Research

Open Access

\title{
Protein C: a potential biomarker in severe sepsis and a possible tool for monitoring treatment with drotrecogin alfa (activated)
}

\author{
Andrew F Shorr ${ }^{1}$, David R Nelson², Duncan LA Wyncoll ${ }^{3}$, Konrad Reinhart ${ }^{4}$, Frank Brunkhorst ${ }^{4}$, \\ George Matthew Vail ${ }^{2}$ and Jonathan Janes ${ }^{2}$
}

\begin{abstract}
1Department of Medicine, Section of Pulmonary and Critical Care Medicine, Washington Hospital Center, Irving Street, Washington, District of Columbia 20010, USA

${ }^{2}$ Lilly Research Laboratories, Eli Lilly and Company, 520 S. Meridian, Indianapolis, Indiana 46285, USA

3Department of Critical Care, Guy's and St Thomas' NHS Foundation Trust, Lambeth Palace Road, London SE1 7EH, UK

${ }^{4}$ Department of Anesthesiology and Intensive Care, Friedrich Schiller University, Erlanger Allee, Jena 07740, Germany

Corresponding author: Andrew F Shorr, afshorr@dnamail.com
\end{abstract}

Received: 19 Nov 2007 Revisions requested: 9 Jan 2008 Revisions received: 13 Feb 2008 Published: 4 Apr 2008

Critical Care 2008, 12:R45 (doi:10.1186/cc6854)

This article is online at: http://ccforum.com/content/12/2/R45

(c) 2008 Shorr et al.; licensee BioMed Central Ltd.

This is an open access article distributed under the terms of the Creative Commons Attribution License (http://creativecommons.org/licenses/by/2.0), which permits unrestricted use, distribution, and reproduction in any medium, provided the original work is properly cited.

\begin{abstract}
Introduction Drotrecogin alfa (activated; DrotAA) treatment, a 96-hour infusion, reduces 28-day mortality in severe sepsis to approximately $25 \%$. The question remains whether a longer infusion or higher dose could increase rate of survival. The goal of this study was to identify a dependable, sensitive measure with which to monitor disease progression and response in patients during DrotAA treatment.
\end{abstract}

Methods Data on severe sepsis patients included in PROWESS (placebo-controlled, double-blind, randomized study of 850 DrotAA and 840 placebo individuals) and ENHANCE (single-arm, open-label study of 2,375 DrotAA patients) studies were analyzed. In these studies, DrotAA (24 $\mu \mathrm{g} / \mathrm{kg}$ per hour) or placebo was infused for 96 hours and patients were followed for 28 days. Data on six laboratory measures and five organ dysfunctions were systematically analyzed to identify a potential surrogate end-point for monitoring DrotAA therapy and predicting 28-day mortality at the end of therapy. To allow comparison across variables, sensitivity and specificity analyses identified cut-off values for preferred outcome, and relative risks for being above or below cut-offs were calculated, as was the 'proportion of treatment effect explained' (PTEE) to identify biomarkers that contribute to benefit from DrotAA.
Results Protein C was the only variable that correlated with outcome across all analyses. Using placebo data, a baseline protein C under $40 \%$ was established as a useful predictor of outcome (odds ratio 2.12). Similar odds ratios were associated with cut-off values of other biomarkers, but the treatment benefit associated with DrotAA was significantly greater below the cutoff than above the cut-off only for protein $C$ (relative risk for 28day mortality 0.66 versus $0.88 ; P=0.04$ ). Protein $\mathrm{C}$ was the only end-of-infusion biomarker that potentially explained at least $50 \%$ of the benefit from DrotAA (PTEE 57.2\%). The PTEE was $41 \%$ for cardiovascular Sequential Organ Failure Assessment score and for d-dimer. At the end of infusion (day 4), protein $\mathrm{C}$ categories $(\leq 40 \%, 41 \%$ to $80 \%$, and $>80 \%$ ) remained significantly related to mortality, regardless of treatment assignment.

Conclusion Based on systematic analyses of 11 variables measured in severe sepsis clinical trials, protein $C$ was the only variable consistently correlated with both DrotAA treatment effect and survival. Further study is needed to determine whether longer infusions or higher doses of DrotAA would achieve the goal of normalizing protein $C$ in more patients with severe sepsis.

\section{Introduction}

Biomarkers play an important role in clinical care [1,2]. Biomarkers facilitate diagnosis, aid in assessing the severity of dis- ease, and provide clinicians with surrogates that they can follow to assess response to therapy. In a number of areas, biomarkers are critical in the management of complex disease

DrotAA = drotrecogin alfa (activated); ENHANCE = Extended Evaluation of Recombinant Activated Protein C; IL = interleukin; IQR = interquartile range; $\mathrm{LOCF}=$ last observation carried forward; $\mathrm{PC}=$ protein $\mathrm{C} ; \mathrm{PROWESS}=$ Recombinant Human Activated Protein C Worldwide Evaluation in Severe Sepsis; PTEE = proportion of treatment effect explained; SOFA = Sequential Organ Failure Assessment. 
states. For example, brain natriuretic peptide is now routinely measured in patients suspected of having decompensated congestive heart failure $[3,4]$, whereas $d$-dimer is evaluated to exclude the diagnosis of venous thromboembolism $[5,6]$. For biomarkers to prove useful, they must be easy to measure, perform well as diagnostic tools, and exhibit some correlation with outcomes. Additionally, biomarkers can serve as surrogate markers in clinical trials. They have been incorporated into studies with the aim being to identify patients who might be eligible for certain experimental interventions and exclude those who are unlikely to benefit from a proposed novel treatment [7].

Severe sepsis and septic shock pose diagnostic challenges because many of the signs and symptoms in these conditions are nonspecific [8]. There is a pressing need to identify a biomarker that correlates with outcomes and that stratifies patients regarding the likelihood that they will benefit from novel therapies such as drotrecogin alfa (activated; DrotAA). Recently a sepsis definitions consensus conference [9] added specific biomarkers to the list of diagnostic criteria for sepsis.

Protein $\mathrm{C}(\mathrm{PC})$ is a vitamin $\mathrm{K}$ dependent plasma serine protease zymogen that is converted to activated $\mathrm{PC}$ by the thrombin-thrombomodulin complex. Activated PC has anticoagulant, anti-inflammatory, cytoprotective, and antiapoptotic activities [10-14].

PC deficiency is prevalent in severe sepsis, with studies showing that more than $80 \%$ of patients with severe sepsis have a baseline PC level below the lower limit of normal [15-18]. Unlike inflammatory cytokines, which are transiently elevated in severe sepsis, plasma PC levels decrease early in patients who develop severe sepsis, often before clinical symptoms appear, and these levels remain low initially but gradually rise in patients who recover and survive [18-21]. Numerous studies have examined the predictive value of plasma PC levels in sepsis [22-26]. Other studies have confirmed the association between depressed PC levels at baseline and the increased likelihood of adverse outcomes in sepsis, including time on a ventilator, time in the intensive care unit, development of shock, and increased mortality [17,18,20,21,25-33]. Previously reported placebo data from the PROWESS (Recombinant Human Activated Protein C Worldwide Evaluation in Severe Sepsis) trial showed that baseline PC levels and early changes in PC were prognostic of outcome. Change in PC levels on the first day after diagnosis of severe sepsis was highly correlated with outcome, with a decrease during the first days being able to differentiate eventual survivors from nonsurvivors [34]. However, broader reliance on PC as a biomarker in severe sepsis and septic shock requires evidence that serial changes over multiple time points provide valuable clinical information. Furthermore, it is necessary to demonstrate that measurement of PC provides information and insight not otherwise available from other biomarkers.
In order to validate the role of PC as a biomarker in severe sepsis and septic shock, we performed a secondary analysis of two large clinical trials of DrotAA. We compared the explanatory power of PC with those of multiple other clinical measures and biomarkers to determine the independent contribution that serial PC measurement would make in explaining mortality and DrotAA response.

\section{Materials and methods \\ Patients}

The PROWESS and ENHANCE (Extended Evaluation of Recombinant Activated Protein C) trials were conducted (before assignment of trial registration numbers) in accordance with ethical principles that have their origin in the Declaration of Helsinki and are consistent with good clinical practice and applicable laws and regulations. The trial designs, patient disposition, inclusion/exclusion criteria, and results were described previously $[15,35]$. PROWESS was a randomized, placebo-controlled clinical trial of DrotAA (Xigris ${ }^{\circledR}$; Eli Lilly and Company, Indianapolis, IN, USA) in adult patients with severe sepsis. ENHANCE was an open-label, single-arm, clinical trial of DrotAA. All investigative sites obtained approval for the study from their institutional review board. Written informed consent was obtained from all patients or their legal representatives.

\section{Biomarker evaluations}

In the PROWESS trial, plasma samples were obtained at baseline (day of randomization) and daily through to study day 7. A central laboratory (Covance Central Laboratory Services, Indianapolis, IN, USA) performed all assays. The PC activity assay was performed on a STA ${ }^{\circledR}$ coagulation analyzer using the STA ${ }^{\circledR}$-Staclot ${ }^{\circledR}$ Protein C kit (Diagnostica Stago, AsnieresSur-Seine, France), which has a coefficient of variation of $7.5 \%$. Protein S measurements were performed on the STA ${ }^{\circledR}$ coagulation analyzer using the STA ${ }^{\circledR}$ - Staclot ${ }^{\circledR}$ Protein $S$ kit (Diagnostica Stago). Antithrombin III activity was quantitated using a chromogenic activity assay (Stachrome ATIII; Diagnostica Stago). IL-6 antigen levels were measured by enzyme immunoassay (Quantikine Human IL-6 HS kit; R\&D Systems, Minneapolis, MN, USA). PC measurements during the ENHANCE trial were obtained at baseline and the end of infusion, and were analyzed using the same methodology as in PROWESS.

Sequential Organ Failure Assessment (SOFA) scores were determined based on local laboratory data, vasopressor dosages, and need for mechanical ventilation.

\section{Statistical methods}

The statistical methods were designed to examine individually each laboratory and clinical measure for their attributes as biomarkers. Biomarkers have been classified into types by the National Institutes of Health Biomarker Definition Working Group [1]. Vasan [2] adapted the National Institutes of Health 
definitions to categorize biomarkers into type 0,1 , and 2; the definition of each type is given below. The following statistical tests examined each type of biomarker using data from the PROWESS trial. Data from the ENHANCE trial, in which all patients received DrotAA, were used to explore the consistency of findings; no combined analyses of the PROWESS and ENHANCE data were performed.

\section{Type 0 biomarker}

A type 0 biomarker is, 'A marker of the natural history of a disease and correlates longitudinally with known clinical indices.' Initial analyses determined which of six laboratory measures and five organ dysfunctions (SOFA scores) were related at baseline to the clinical index of 28-day mortality in the placebo group. Based on placebo patients $(n=840)$, an 'optimal' cutoff was generated that maximized the sum of sensitivity and specificity (with each required to be at least 40\%) to predict 28-day mortality. All values across the range of the receiver operating characteristic curves were examined. Using a cut-off for each measure allowed comparisons of odds ratios and interactions with treatment on a consistent binary scale across measures. In addition, these same measures at day 4 were evaluated for the placebo patients. Significance at both time points using the significance of $\chi^{2}$ tests and 95\% confidence intervals of odds ratios would indicate longitudinal correlation with mortality.

\section{Type 1 biomarker}

A type 1 biomarker is, 'A marker that captures the effects of a therapeutic intervention in accordance with its mechanism of action.' This was examined in two ways for DrotAA in PROWESS. First, do more severe baseline values for the biomarker indicate a subgroup with a greater treatment benefit? This statistical interaction between biomarker and treatment was tested with Breslow-Day tests. The relative risks for death on comparing DrotAA $(n=850)$ with placebo $(n=840)$ were generated above and below cut offs. Second, biomarkers were identified that improved during treatment. Wilcoxon ranksum tests were used to identify laboratory values and organ dysfunctions that were significantly different at day 4 between DrotAA and placebo patients. Day 4 last observation carried forward (LOCF) values were used in these analyses, with no imputation for death (the last observed SOFA score, not '4', was used for patients who died during the first 4 days). Patients with missing baseline values were excluded from these analyses.

\section{Surrogate end-point (type 2 biomarker)}

A type 2 biomarker is, 'A marker that is intended to substitute for a clinical endpoint; a surrogate endpoint is expected to predict clinical benefit.' To quantify the potential of surrogate markers at the end of infusion, methods proposed by $\mathrm{Li}$ and coworkers [36] were utilized using Day 4 values. These methods use logistic regression to provide the 'proportion of treatment effect explained' (PTEE). PTEE has been proposed as a measure of surrogacy for the validation of surrogate endpoints. A good surrogate marker accounts for a larger percentage of treatment effect. For instance, if a treatment reduces the risk for death by $20 \%$ and improvement in a biomarker was associated with a risk reduction of death by $10 \%$, then the biomarker explains $50 \%$ of the treatment effect. This was quantified by taking the ratio of risk reduction explained solely by the average change in a measure, and dividing by total risk reduction associated with the average change in a measure plus the residual treatment effect. These analyses were to determine how much of the 28-day mortality effect was accounted for solely by patient status on day 4 . The PTEE values of the multiple variables examined are not expected to add up to $100 \%$, and a negative PTEE means that the treatment resulted in a change in the variable that is in the opposite direction than anticipated for a beneficial treatment effect.

\section{Additional statistical methods}

Additional nonparametric analyses were performed using Wilcoxon sign-rank and Wilcoxon rank-sum tests, as appropriate. All calculations were performed using SAS version 8.1 software (SAS Institute Inc., Cary, NC, USA).

\section{Results}

The baseline characteristics for the PROWESS placebo and DrotAA patient populations have been reported elsewhere [15], as have those of the ENHANCE population [35]. However, a summary of selected baseline characteristics that are specifically relevant to the present analyses is given in Table 1.

\section{Type 0 biomarker: relationship to natural history of sepsis and correlated with clinical outcome}

Baseline values of six laboratory and five clinical measures were evaluated as potential predictors of 28-day mortality. To allow comparisons across measures, the cut-off values associated with greater risk for mortality based on sensitivity and specificity analyses of baseline values were determined (Table 2). The number of patients at increased risk based on the cutoffs, although each representing a different subgroup, was very similar across variables, representing approximately onethird of patients. However, this does not represent the same high-risk patients in each group. Only one patient was high risk for all 11 markers, and only 48 (5.8\%) were low risk for all of their measures. This approach established a baseline PC level $<40 \%$ as a useful end-point for assessing mortality risk in sepsis patients. The odds of dying within 28 days was twice as high in patients with a baseline PC level $<40 \%$ as in those with a $P C$ level of $\geq 40 \%$. Similar odds ratios were associated with the cut-off values of the other variables, as were the areas under the receiver operating characteristic curve, a combined measure of sensitivity and specificity. This analysis also demonstrates (as already known) the unequal effect of individual SOFA scores, with cut-off ranging from $\geq 1$ for renal SOFA to $\geq 4$ for cardiovascular and respiratory SOFA. 
Table 1

PROWESS and ENHANCE patient baseline characteristics

\begin{tabular}{|c|c|c|c|}
\hline \multirow[t]{2}{*}{ Variable } & \multicolumn{2}{|c|}{ PROWESS } & \multirow{2}{*}{$\begin{array}{c}\text { ENHANCE } \\
\text { DrotAA }(n=2378)\end{array}$} \\
\hline & Placebo $(n=840)$ & $\operatorname{Drot} A A(n=850)$ & \\
\hline Sex (\% male $[n])$ & $58.0(487)$ & $56.1(477)$ & $58.2(1383)$ \\
\hline Mean age (years [SD]) & $60.6(16.5)$ & $60.5(17.2)$ & $59.1(16.9)$ \\
\hline Caucasian $(\%[n])$ & $82.0(689)$ & $81.8(695)$ & $90.6(2154)$ \\
\hline APACHE II score (mean [SD]) & $25.0(7.8)$ & $24.6(7.6)$ & $22.0(7.4)$ \\
\hline \multicolumn{4}{|l|}{ SOFA score (mean [SD]) } \\
\hline Cardiovascular & $2.7(1.5)$ & $2.6(1.5)$ & $3.0(1.4)$ \\
\hline Respiratory & $2.7(1.1)$ & $2.7(1.0)$ & $2.7(1.0)$ \\
\hline Renal & $1.1(1.1)$ & $1.1(1.1)$ & $1.3(1.2)$ \\
\hline Hematologic & $0.7(1.0)$ & $0.7(0.9)$ & $0.8(1.0)$ \\
\hline Hepatic & $0.6(0.9)$ & $0.6(0.8)$ & $0.7(0.9)$ \\
\hline Protein C (median [IQR]) & 50 (33 to 68 ) & 47 (30 to 63$)$ & 45 (30 to 64$)$ \\
\hline Protein S (median [IOR]) & 38 (23 to 58$)$ & 35 (33 to 57 ) & - \\
\hline Antithrombin III (median [IQR]) & 60 (45 to 75$)$ & 58 (43 to 74$)$ & - \\
\hline Interleukin-6 (median [IQR]) & 484 (129 to 2539$)$ & 496 (153 to 2701$)$ & - \\
\hline Prothrombin time (median [IQR]) & 18.6 (16.4 to 21.8$)$ & 18.7 (16.6 to 22.1$)$ & - \\
\hline D-dimer (median [IOR]) & $4.1(2.2$ to 8.7$)$ & $4.2(2.3$ to 8.1$)$ & - \\
\hline
\end{tabular}

APACHE, Acute Physiology and Chronic Health Evaluation; DrotAA, drotrecogin alfa (activated); ENHANCE, Extended Evaluation of Recombinant Activated Protein C; PROWESS, Recombinant Human Activated Protein C Worldwide Evaluation in Severe Sepsis; SD, standard deviation; SOFA, Sequential Organ Failure Assessment; IQR, interquartile range.

To determine which measures exhibited a longitudinal correlation with mortality, these same measures were evaluated at the end-of-infusion period (day 4) for placebo patients. The optimal cut-off values at day 4, shown in Table 3, were very similar to those shown for baseline values in Table 2, except that the cut-off value for IL- 6 was $\geq 185.6$ versus $\geq 704.6 \mathrm{pg} / \mathrm{ml}$. Without adjusting for baseline values, the day 4 values for all 11 variables were associated with a statistically significant increased risk for death at day 28.

\section{Type 1 biomarker: therapeutic intervention in accordance with mechanism of action}

Figure 1 shows the therapeutic effect of DrotAA in patients at lower and higher risk for death, as defined by the statistically defined baseline cut-off for the 11 potential biomarkers shown in Table 2. PC was the only biomarker at baseline that exhibited a statistically significant difference in relative risk for death between the lower and higher risk groups (relative risk 0.66 [DrotAA and placebo] for lower risk versus 0.88 for higher risk patients; $P=0.04)$. In PROWESS, patients who had values below the PC cut-off $(<40 \%)$ and who were administered DrotAA exhibited a $34 \%$ reduction in risk for death $(27.6 \%$ DrotAA versus $41.8 \%$ placebo), whereas above the cut-off the risk reduction was $12 \%$ (22.4\% versus $25.3 \%$ ). Mortality rates observed in the ENHANCE trial were $33.3 \%$ for patients with
PC below the cut-off and $17.6 \%$ for those with PC above the cut-off (data not shown).

\section{Surrogate endipoint (type 2 biomarker): substitute for clinical end-point by predicting clinical benefit}

The next step was to determine which of the potential biomarkers improved during DrotAA treatment (Table 4). In PROWESS, at the end of the 4-day infusion (day 4) DrotAA significantly increased the median PC level $(P<0.0001)$, increased prothrombin time $(P=0.0003)$ and decreased $d$ dimer $(P<0.0001)$, and, to a lesser degree, decreased the cardiovascular SOFA score $(P=0.01)$ and increased the hepatic SOFA score $(P=0.04)$. Although all of these postbaseline measures were prognostic for placebo mortality (Table 3), the end of infusion (day 4 LOCF) level of PC and, to a lesser degree, cardiovascular dysfunction and d-dimer appeared to be specifically improved with DrotAA treatment. In PROWESS, the median increase in PC activity during the 4-day infusion period was 19\% (interquartile range [IOR] 3\% to $36 \%$ ) for DrotAA, as compared with $8 \%$ (IOR $-5 \%$ to $+25 \%$ ) for placebo patients. In the same timeframe, ENHANCE patients receiving DrotAA exhibited an $18 \%$ increase in PC (IOR 0\% to 39\%). Because the negative relationship of DrotAA treatment with hepatic SOFA on day 4 , we reviewed the actual baseline and day 4 bilirubin measure- 
Table 2

\begin{tabular}{|c|c|c|c|c|}
\hline Baseline measure & Cut-offa & Number of patients at increased risk using cut-off (n [\%]) & Odds ratio $(95 \% \mathrm{Cl})$ & $A \cup C^{b}$ \\
\hline Protein C (\%) & $<40$ & $243(31.4 \%)$ & $2.12(1.55-2.89)$ & $58.9 \%$ \\
\hline Protein S (\%) & $<46$ & $239(31.5 \%)$ & $1.91(1.38-2.64)$ & $57.7 \%$ \\
\hline Antithrombin III (\%) & $<53$ & $240(31.4 \%)$ & $2.32(1.70-3.18)$ & $60.1 \%$ \\
\hline interleukin-6 (pg/ml) & $\geq 704.6$ & $252(31.2 \%)$ & $2.21(1.63-2.99)$ & $59.7 \%$ \\
\hline Prothrombin time (seconds) & $\geq 18.4$ & $240(31.5 \%)$ & $1.89(1.38-2.58)$ & $57.4 \%$ \\
\hline D-dimer $(\mu \mathrm{g} / \mathrm{ml})$ & $\geq 4.45$ & $241(31.8 \%)$ & $1.51(1.11-2.05)$ & $55.1 \%$ \\
\hline Cardiovascular SOFA & $\geq 4$ & $259(30.8 \%)$ & $1.63(1.21-2.18)$ & $56.0 \%$ \\
\hline Respiratory SOFA & $\geq 4$ & $257(31.2 \%)$ & $1.76(1.27-2.44)$ & $55.5 \%$ \\
\hline Renal SOFA & $\geq 1$ & $258(30.8 \%)$ & $2.14(1.55-2.95)$ & $58.6 \%$ \\
\hline Hematologic SOFA & $\geq 2$ & $259(30.8 \%)$ & $1.69(1.20-2.38)$ & $54.6 \%$ \\
\hline Hepatic SOFA & $\geq 2$ & 239 (31.3\%) & $1.31(0.89-1.93)$ & $52.1 \%$ \\
\hline
\end{tabular}

aCut-off based on maximum sensitivity and specificity when both were $\geq 40 \%$ for predicting 28 -day mortality. Using a cut-off for each measure allowed comparison of odds ratios and treatment interactions on a consistent binary scale across variables. bArea under the receiver operating characteristic curve (AUC) based on 28-day mortality outcome in logistic regression models with the cut-off as the univariate independent variable; this is a combined measure of sensitivity and specificity. Cl, confidence interal; PROWESS, Recombinant Human Activated Protein C Worldwide Evaluation in Severe Sepsis; SOFA, Sequential Organ Failure Assessment.

ments. Considering the 1,374 patients in PROWESS for whom data were available regarding the change in bilirubin from baseline at these time points $(n=692$ for DrotAA and $n$ $=682$ for placebo), there were no significant changes within groups $(P=0.49$ for DrotAA and $P=0.12$ for placebo) or between therapies $(P=0.14)$, with a median change of 0 and $-1 \mu \mathrm{mol} / / \mathrm{l}$ for DrotAA and placebo, respectively.

To assess how the change helps to account for the DrotAA treatment effect in PROWESS, the PTEE was analyzed (Table 4). The end-of-infusion (day 4 LOCF) measure of PC accounted for $57.2 \%$ of the DrotAA treatment effect on 28 day mortality. The change in prothrombin time and the hepatic SOFA, while being statistically different between treatment groups, exhibited a negative correlation with the DrotAA treatment effect (namely, its direction was opposite that anticipated for a beneficial treatment effect).

\section{Further examination of protein $C$ and DrotAA interactions over time}

A strong link between improved PC levels and improved survival became apparent when serial PC levels were analyzed for DrotAA treated patients and displayed by time of death or ultimate survival (Figure 2a). (A similar figure for the PROWESS placebo patients was previously reported [22].) As with the PROWESS placebo patients, PC levels assessed at the start of each time interval were highly predictive of outcome within the time interval, with continued PC deficiency associated with higher mortality. This analysis demonstrates that patients with lower PC levels are more likely to die, and that patients who survived to be discharged had $\mathrm{PC}$ levels that increased during the DrotAA infusion to a mean of $80 \%$ by day 5 . Patients who died between days 6 and 15 had a decrease in mean PC levels after day 4, suggesting that PC levels fell when DrotAA infusion was stopped.

Based on the statistical analyses presented in Table 4, d-dimer values also appeared to be a potential surrogate biomarker for DrotAA therapy. However, as shown in Figure $2 b$, although $d$ dimer decreased in all patients who received DrotAA, at the end of the infusion the $d$-dimer levels immediately began to increase in all patients and that increase was not correlated with mortality at different time points.

\section{Summary of results correlated with biomarker status}

To aid in the interpretation of these data, the results are summarized in Table 5 using categories defined in the footnote. This summary shows that PC is the only biomarker that consistently correlated with outcome, regardless of the time of measurement or the analytical approach.

Figure 3 shows that PC levels at end of infusion also correlated with outcome regardless of treatment. For this final analysis, end-of-infusion (day 4 LOCF) PC levels were categorized by deficiency (severe $\leq 40 \%$, moderate $41 \%$ to $80 \%$, or normal $>80 \%$ ) and the categories were shown to be significantly related to mortality regardless of treatment. DrotAA treatment resulted in fewer patients (166 [20.8\%] versus 217 [28.0\%]) with severe PC deficiency $(\leq 40 \%)$, and more patients $(290$ [36.3\%] versus 211 [27.2\%]) with normal PC levels (> 80\%) at the end of infusion compared with placebo $(P<0.0001)$. The ENHANCE mortality rates based on day 4 PC categories were consistent with the PROWESS DrotAA data. Regardless of treatment, mortality rates were lowest in patients with 
Table 3

Relationship of day 4 (end of infusion) values to 28-day mortality in PROWESS placebo patients

\begin{tabular}{|c|c|c|c|c|c|}
\hline \multirow[b]{2}{*}{ Measure } & \multirow[b]{2}{*}{ Cut-offa } & \multicolumn{2}{|c|}{ Sample size } & \multicolumn{2}{|c|}{ Univariate odds ratio } \\
\hline & & Higher risk & Lower risk & Odds ratio $(95 \% \mathrm{Cl})$ & $P$ \\
\hline Protein C (\%) & $<42$ & 240 & 578 & 4.63 (3.35 to 6.41$)$ & $<0.0001$ \\
\hline Protein S (\%) & $<42$ & 297 & 521 & 3.25 (2.38 to 4.43$)$ & $<0.0001$ \\
\hline Antithrombin III (\%) & $<60$ & 306 & 512 & 4.17 (3.05 to 5.71$)$ & $<0.0001$ \\
\hline Interleukin-6 (pg/ml) & $\geq 185.6$ & 264 & 563 & 7.27 (5.23 to 10.11$)$ & $<0.0001$ \\
\hline Prothrombin time (seconds) & $\geq 18.4$ & 233 & 585 & $6.13(4.40$ to 8.56$)$ & $<0.0001$ \\
\hline D-dimer $(\mu \mathrm{g} / \mathrm{ml})$ & $\geq 4.63$ & 408 & 410 & 2.13 (1.57 to 2.89 ) & $<0.0001$ \\
\hline Cardiovascular SOFA & $\geq 3$ & 301 & 539 & 7.32 (5.28 to 10.13$)$ & $<0.0001$ \\
\hline Respiratory SOFA & $\geq 3$ & 358 & 482 & 3.36 (2.48 to 4.56$)$ & $<0.0001$ \\
\hline Renal SOFA & $\geq 1$ & 335 & 505 & 4.82 (3.52 to 6.59$)$ & $<0.0001$ \\
\hline Hematologic SOFA & $\geq 2$ & 252 & 588 & 3.42 (2.50 to 4.68$)$ & $<0.0001$ \\
\hline Hepatic SOFA & $\geq 1$ & 256 & 573 & 2.11 (1.55 to 2.89 ) & $<0.0001$ \\
\hline
\end{tabular}

aCut-off was defined by the day 4 (end of infusion) value that resulted in maximum sensitivity and specificity for predicting 28-day mortality. Cl, confidence interal; PROWESS, Recombinant Human Activated Protein C Worldwide Evaluation in Severe Sepsis; SOFA, Sequential Organ

Failure Assessment.

normalized PC levels. The histograms within each PC category appeared equal across treatments, indicating that differences between treatment groups do not exist after taking into

Figure 1

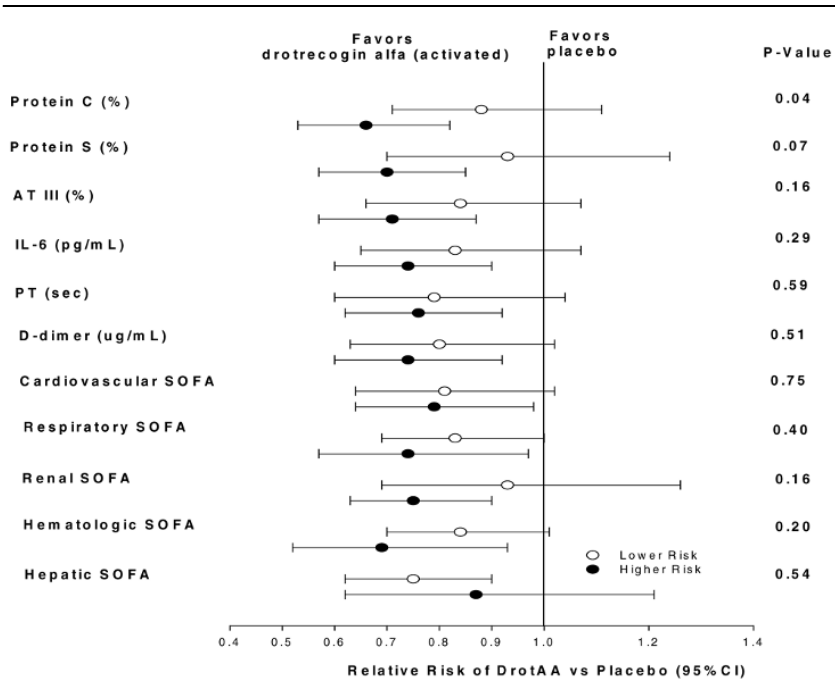

Illustration of 28-day mortality RR reduction (DrotAA versus placebo) for each potential biomarker at baseline. The point estimates of relative risk (RR) for death in patients at lower risk and higher risk, based on statistically defined cut-offs (shown in Table 2), are indicated by open ovals and solid ovals, respectively; $95 \%$ confidence intervals (Cls) are indicated by horizontal lines. Only protein C (PC) was significantly $(P<$ 0.05 ) different between the two risk groups, as indicated by the least overlap in Cls, indicating a differential benefit. $P$ values were determined using Breslow-Day tests. AT, antithrombin; $\mathrm{Cl}$, confidence interval; DrotAA, drotrecogin alfa (activated); IL, interleukin; PT, prothrombin time; SOFA, Sequential Organ Failure Assessment. account the surrogate end-point. The end-of-infusion PC category has a greater effect on mortality than differences within a category between DrotAA and placebo treatment.

\section{Discussion}

These analyses indicate that PC can act as a surrogate endpoint in severe sepsis, regardless of the time of measurement or treatment received. Based on a systematic statistical assessment of six potential biomarkers and five organ dysfunction measures that were measured in a large randomized clinical trial conducted in severe sepsis patients, only PC levels were significantly correlated with 28 -day mortality regardless of statistical approach. Using statistically defined cut-offs, multiple variables at baseline were predictive of 28-day mortality and all variables could be predictive at the end of the 4-day infusion. Only PC improved with DrotAA treatment, was significantly correlated with the DrotAA treatment effect, and accounted for more than $50 \%$ of its treatment effect (PTEE). Additionally, serial changes in PC correlated well with mortality. Variations in PC also explained the majority of the treatment effect due to DrotAA therapy.

Other biomarkers have been proposed to be useful diagnostic markers for sepsis and the severity of sepsis [37], but those analyzed in this study did not meet all criteria for surrogacy. Ddimer did decrease during infusion with DrotAA and, based on PTEE analysis, it could account for $41 \%$ of its treatment effect, but there was no difference in relative risk between the lower and higher risk groups. At the end of infusion the values increased in all patients, both survivors and nonsurvivors, suggesting that the DrotAA effect is not just an alteration in the procoagulant state. Instead, based on PROWESS data, d- 
Table 4

Day 4 (end of infusion) values in PROWESS:individual surrogate performance score (PTEE)

\begin{tabular}{lcccc}
\hline Day 4 measure & $\begin{array}{c}\text { DrotAA patients } \\
\text { (mean [SD]/median) }\end{array}$ & $\begin{array}{c}\text { Placebo patients } \\
\text { (mean [SD]/median) }\end{array}$ & $P^{\mathrm{a}}$ & Individual surrogate performance score (PTEE) \\
\hline Protein C (\%) & $70.6(36.2) / 67.0$ & $62.7(36.9) / 59.0$ & $<0.0001$ & $57.2 \%$ \\
Protein S (\%) & $53.3(29.0) / 52.0$ & $53.5(30.2) / 53.0$ & 0.95 & $-0.8 \%^{\mathrm{c}}$ \\
Antithrombin III (\%) & $70.3(27.0) / 69.5$ & $69.0(28.0) / 70.0$ & 0.38 & $13.4 \%$ \\
Interleukin-6 (pg/ml) & $3,649(30,280) / 75.5$ & $4948(33379) / 79.5$ & 0.78 & $0.3 \% \%^{\mathrm{d}}$ \\
Prothrombin time (seconds) & $18.6(7.4) / 16.7$ & $18.5(8.4) / 16.2$ & 0.0003 & $-30.4 \% \%^{\mathrm{c}, \mathrm{d}}$ \\
D-dimer ( $\mu \mathrm{g} / \mathrm{ml})$ & $4.63(5.41) / 3.12$ & $7.13(7.87) / 4.61$ & $<0.0001$ & $40.5 \% 0^{\mathrm{d}}$ \\
Cardiovascular SOFA & $1.48(1.60) / 1.00$ & $1.67(1.64) / 1.00$ & 0.01 & $40.8 \%$ \\
Respiratory SOFA & $2.25(1.03) / 2.00$ & $2.30(1.01) / 2.00$ & 0.25 & $12.7 \%$ \\
Renal SOFA & $0.74(1.12) / 0.00$ & $0.80(1.17) / 0.00$ & 0.39 & $10.6 \%$ \\
Hematologic SOFA & $0.89(1.12) / 0.00$ & $0.92(1.15) / 0.00$ & 0.79 & $5.1 \%$ \\
Hepatic SOFA & $0.61(0.92) / 0.00$ & $0.53(0.89) / 0.00$ & 0.04 & $-13.6 \%$ \\
\hline
\end{tabular}

a $P$ value for Wilcoxon rank-sum test. ${ }^{b}$ The performance score shows the proportion of drotrecogin alfa (activated; DrotAA) treatment effect explained (PTEE) based on logistic regression analyses that quantify the amount of the observed treatment effect on 28-day mortality that is attributable to the treatment effect of the individual biomarker. Because changes in these biomarkers are often interdependent (for example, protein $\mathrm{C}$ and cardiovascular SOFA improvements), the performance scores are not expected to add up to $100 \%$. ${ }^{\mathrm{C}} \mathrm{A}$ negative performance score means that the treatment adversely affected the variable. $\mathrm{d}$ For biomarkers that varied greatly between mean and median values, analysis was based on median value. PROWESS, Recombinant Human Activated Protein C Worldwide Evaluation in Severe Sepsis; SD, standard deviation; SOFA, Sequential Organ Failure Assessment.

dimer appears to be a marker that the patient received DrotAA, not how well the patient responded to DrotAA. IL-6 levels at baseline were predictive of patient outcome, but at the end of infusion (day 4) the difference between DrotAA and placebo groups was not significant and its PTEE was $0.3 \%$. Prothrombin is another biomarker that is expected to decrease as coagulopathy improves, but instead prothrombin time increased slightly, but consistently, in patients who received DrotAA, resulting in a negative PTEE value $(-30 \%)$. Although DrotAA improves coagulopathy, there is also a small direct effect of interference of activated PC with prothrombin time. The negative PTEE obtained for hepatic SOFA (-14\%) results from a slightly higher hepatic SOFA with DrotAA than with placebo. However, there were no significant differences in change in bilirubin from baseline to day 4. Traditionally, liver dysfunction under conditions of shock and sepsis is considered to be biphasic, with an initial ischaemic insult (ischaemic hepatitis) followed by jaundice (intensive care unit jaundice) developing several days later $[38,39]$. Thus, the SOFA subscore for hepatic impairment, which is based on bilirubin levels, is biased toward underestimation of dysfunction in the early course of the disease.

Some have expressed skepticism toward PTEE analyses, especially when it is applied to small individual studies, because high PTEE values do not necessarily imply that the surrogate end-point is an important part of the causal pathway that leads from treatment to disease [40]. However, our analyses are based on a large population $(n=1,690)$ and show that $\mathrm{PC}$ levels not only can explain a large proportion of the DrotAA treatment effect but also are directly related to clinical outcome in severe sepsis.

PC meets the US National Institutes of Health's recommended definition of a biomarker that could function as a clinical endpoint (a variable that reflects how long or how a patient feels or functions, or how long a patient survives), as well as a surrogate end-point (a biomarker, based on epidemiologic, therapeutic, pathophysiologic, or other scientific evidence, intended to substitute for a clinical end-point) [40]. Surrogate end-points can be useful in advising patients about modifications of treatment after they have reached a surrogate endpoint but have not yet reached the true clinical end-point. Our data shows $\mathrm{PC}$ to be a valid surrogate, defined as a biomarker that can explain at least $50 \%$ of the effect of an exposure or intervention on the outcome of interest [41]. Of the biomarkers analyzed, only PC had a PTEE greater than 50\%. The PTEE for cardiovascular SOFA was $41 \%$. PC predicts cardiovascular changes downstream, and so it is expected that the cardiovascular SOFA would be a reasonable surrogate. However, the reverse is not true; cardiovascular improvement does not necessarily increase PC downstream, and the baseline cardiovascular SOFA does not appear to predict well who will benefit most from DrotAA treatment. Although this is somewhat counterintuitive, it is what the PROWESS data have indicated.

Normalization of PC levels is critical for survival. The serial measurements of PC show that if the $P C$ values continue to increase toward normalization after day 4, then survival increases. For those patients who died between days 6 and 
Figure 2

(a)

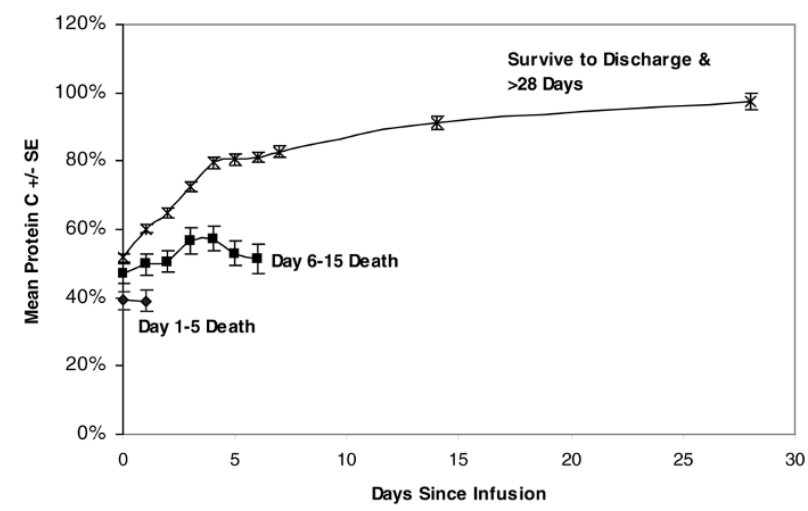

(b)

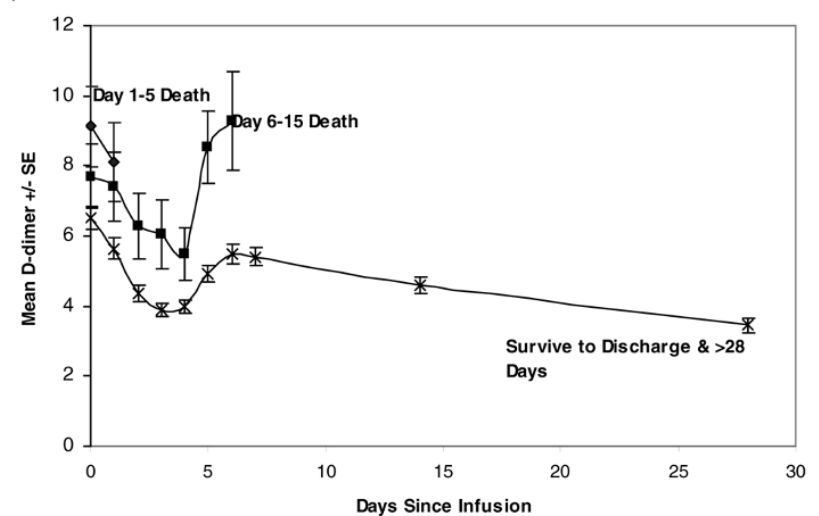

$\mathrm{PC}$ and d-dimer levels. Shown are the mean \pm standard error (a) protein $C(P C)$ and (b) d-dimer levels based on time of death. Raw values with no imputation were included. PROWESS (Recombinant Human Activated Protein C Worldwide Evaluation in Severe Sepsis) drotrecogin alfa (activated; DrotAA) patients with baseline measures were classified according to timing of death $(n=P C / d$-dimer): death $\leq 5$ days after start of infusion $(n=79 / 86)$; death after 6 to 15 days $(n=$ 81/84); and survival to day 28 and hospital discharge $(n=544 / 577)$. The PC data were reported by Vangerow and coworkers [42] and comparable PC data for PROWESS placebo patients were reported by Macias and Nelson [22]

15 , the PC levels were increasing until after the end of drug infusion, which raises the question of whether these patients would have survived if the DrotAA infusion had been extended beyond day 4. Use of PC levels to optimize therapy for individual patients warrants further study. A series of studies are proposed to explore the use of serial plasma PC measurement as a biomarker that will achieve the following objectives: aid in the identification of patients with severe sepsis who are most likely to benefit from DrotAA; enable the adjustment of DrotAA therapy for individual patients (specifically, the possibility to use a higher dose and to adjust the infusion duration, making it either longer or shorter as needed); and provide guidance to the clinician regarding whether the patient is responding to DrotAA. The first study in the series is referred to as RESPOND (Research Evaluating Serial PC Levels in Severe Sepsis Patients on DrotAA) and is currently ongoing. It is seeking to demonstrate that 'alternative therapy' (higher dose with variable infusion duration or variable infusion duration only) results in a greater increase in PC levels than 'standard therapy' (the currently approved regimen of $24 \mu \mathrm{g} /$ kg per hour for 96 hours) and, importantly, to provide appropriate safety and efficacy data to determine the most appropriate aspects of 'alternative therapy' to incorporate into possible future studies [42].

\section{Limitations}

This was a post hoc analysis that was limited to the potential biomarkers measured during PROWESS. When PROWESS was designed the prevailing assumed mechanism of action of PC was anticoagulation, and so the laboratory measurements in that study focused primarily on the coagulation pathway.

Many of the potential biomarkers included in our analyses do not have prespecified clinically defined thresholds. Therefore, to be consistent in how the variables were analyzed, statistically defined cut-offs were determined from specificity and sensitivity analyses. The cut-offs were driven by variability within the patient population in PROWESS and were therefore limited by a one-study dataset. We used ENHANCE data in an attempt to validate our findings, but that comparison is not ideal because ENHANCE had no placebo group and PC was measured less frequently during the trial. Also, the areas under the receiver operating characteristic curves for all markers tended to be at the $60 \%$ level or below. In the PROWESS population, in which the extremes of risk for death are excluded by inclusion and exclusion criteria, individual markers of baseline severity have relatively low values for prognostic measures in univariate analyses.

Finally, in an attempt to put our analyses into perspective and to help summarize the results from the different analyses, we arbitrarily assigned categories to the outcomes, as shown in Table 5. This was an effort to illustrate, not quantitate, the results.

\section{Conclusion}

Based on systematic analyses of 11 variables (six biomarkers and five organ dysfunctions) measured in severe sepsis clinical trials, PC was the only variable consistently correlated with both DrotAA treatment effect and survival. Further study is needed to determine whethter longer infusions or higher doses of DrotAA would achieve the goal of normalizing PC in more patients with severe sepsis.

\section{Competing interests}

DRN, JJ, and GMV are employees of and stockholders in Eli Lilly and Company (Eli Lilly), the manufacturer of DrotAA. AFS is a consultant to both Eli Lilly and Astra Zeneca regarding the design of clinical trials for severe sepsis and septic shock. DLAW has given paid lectures for and participated in clinical trials, supported by Eli Lilly. KR has served as consultant and 
Summary of results in support of biomarker status

\begin{tabular}{|c|c|c|c|c|c|}
\hline & $\begin{array}{l}\text { Type } 0 \text { biomarker: } \\
\text { placebo baseline } \\
\text { value versus mortality } \\
\text { (see Table 2); } \\
\text { categorized by ORa }\end{array}$ & $\begin{array}{l}\text { Type } 0 \text { biomarker: } \\
\text { placebo day } 4 \text { value } \\
\text { versus mortality (see } \\
\text { Table } 3 \text { ); categorized } \\
\text { by } P \text { value }\end{array}$ & $\begin{array}{l}\text { Type } 1 \text { biomarker: } \\
\text { relationship of } \\
\text { baseline value to } \\
\text { DrotAA effect (see } \\
\text { Figure } 1) \text {; categorized } \\
\text { by } P \text { value }\end{array}$ & $\begin{array}{l}\text { Surrogate (type } 2 \\
\text { biomarker): } \\
\text { improvement at day } 4 \\
\text { with DrotAA (see } \\
\text { Table } 4 \text { ); categorized } \\
\text { by } P \text { value }^{\mathrm{b}}\end{array}$ & $\begin{array}{l}\text { Surrogate (type } 2 \\
\text { biomarker): surrogate } \\
\text { performance score } \\
\text { (see Table 4); } \\
\text { categorized by PTEE }\end{array}$ \\
\hline Protein $C$ & +++ & +++ & ++ & +++ & +++ \\
\hline Protein S & ++ & +++ & + & - & - \\
\hline Antithrombin III & +++ & +++ & - & - & + \\
\hline Interleukin-6 & +++ & +++ & - & - & - \\
\hline Prothrombin time & ++ & +++ & - & +++ & - \\
\hline D-dimer & ++ & +++ & - & +++ & ++ \\
\hline Cardiovascular SOFA & ++ & +++ & - & ++ & ++ \\
\hline Respiratory SOFA & ++ & +++ & - & - & + \\
\hline Renal SOFA & +++ & +++ & - & - & + \\
\hline Hematologic SOFA & ++ & +++ & - & - & + \\
\hline Hepatic SOFA & + & +++ & - & + & - \\
\hline
\end{tabular}

Shown is the categorization based on the results of each analysis. To summarize the statistical analyses, the results from each analysis were categorized as follows. aOdds ratios (ORs) from Table $2:-=$ OR $<0 ;+=0 \leq \mathrm{OR}<1.5 ;++=$ OR 1.5 to $2.0 ;+++=$ OR $>2.0$. bP values from Tables 3 and 4 , and Figure $1:-=P>0.1 ;+=0.051<P \leq 0.1 ;++=P 0.01$ to $0.05 ;+++=P<0.01$. cProportion of treatment effect explained (PTEE) from Table $4:-=$ negative or $<5 \% ;+=5 \%$ to $<25 \% ;++=25 \%$ to $50 \% ;+++=>50 \%$. DrotAA, drotrecogin alfa (activated); SOFA, Sequential Organ Failure Assessment.

received payments from Eli Lilly for speaking engagements and research. FB received payments from Eli Lilly for speaking engagements and research.

\section{Figure 3}

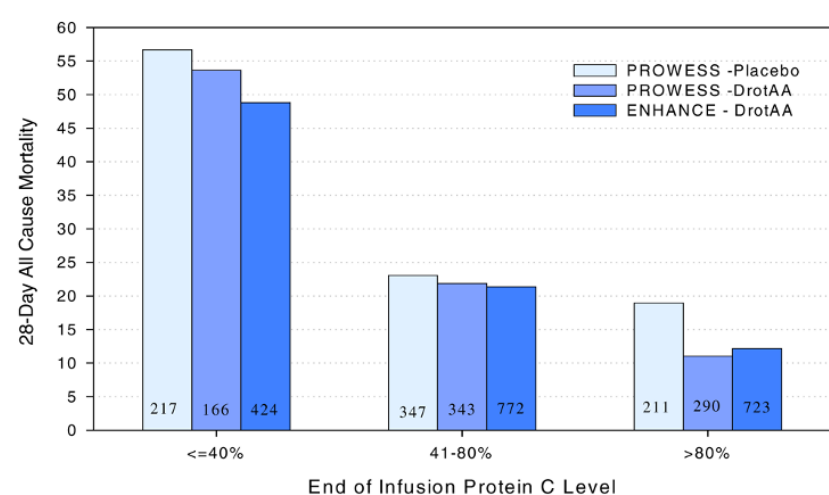

Mortality from PROWESS and ENHANCE based on end-of-infusion $\mathrm{PC}$ levels by categories. The protein $\mathrm{C}(\mathrm{PC})$ categories were normal $(>$ $80 \%)$, deficient $(41 \%$ to $80 \%)$, and severely deficient $(<40 \%)$. The number in each column is the total number of patients in each category. Patients were included if they had a baseline PC measure. Day 4 PC was classified as end of infusion. If day 4 measurement was not available, last observation carried forward values were used for classification. These data were reported by Vangerow and coworkers [42]. ENHANCE, Extended Evaluation of Recombinant Activated Protein C; PROWESS, Recombinant Human Activated Protein C Worldwide Evaluation in Severe Sepsis.

\section{Key messages}

- Serial measurement of PC in sepsis has the potential to act as a biomarker to predict outcome and guide therapy with DrotAA.

- Based on systematic analyses of 11 variables (six biomarkers and five organ dysfunctions) measured in severe sepsis clinical trials, PC was the only variable consistently correlated with both survival and DrotAA treatment effect.

- A PC level $<40 \%$ was established as a useful predictor of outcome at baseline and at the end of infusion.

- Normalization of PC levels is an important predictor of survival, and DrotAA treatment results in more patients with normal PC levels and fewer patients with severe $\mathrm{PC}$ deficiency at the end of infusion compared with placebo.

- Further study is needed to determine whether longer infusions or higher doses of DrotAA would achieve the goal of normalizing PC in more patients with severe sepsis.

\section{Authors' contributions}

AFS, DRN, and JJ participated in the conception and design of the study. AFS, and DLAW participated in the clinical trials and data collection. GMV participated in the conception of the study. All authors contributed to the development and conduct 
of analyses, and participated in drafting the manuscript. All authors contributed to revisions and approval of the final manuscript.

\section{Acknowledgements}

We thank Delores Graham, a contract medical writer, and David Sundin, an Eli Lilly employee, who provided editorial service on behalf of Eli Lilly in preparation of the manuscript, and Chuyun Huang, an Eli Lilly employee, who provided statistical analysis support. The PROWESS and ENHANCE studies, and the statistical analyses for this manuscript, were funded by Eli Lilly.

\section{References}

1. NIH Biomarkers Definitions Working Group: Biomarkers and surrogate endpoints: preferred definitions and conceptual framework. Clin Pharmacol Ther 2001, 69:89-95.

2. Vasan RS: Biomarkers of cardiovascular disease: molecular basis and practical considerations. Circulation 2006, 113:2335-2362.

3. Moe GW: B-type natriuretic peptide in heart failure. Curr Opin Cardiol 2006, 21:208-214.

4. McKie PM, Burnett JC Jr: B-type natriuretic peptide as a biomarker beyond heart failure: speculations and opportunities. Mayo Clin Proc 2005, 80:1029-1036.

5. Qaseem A, Snow V, Barry P, Hornbake ER, Rodnick JE, Tobolic T, Ireland B, Segal JB, Bass EB, Weiss KB, Green L, Owens DK, Joint American Academy of Family Physicians/American College of Physicians Panel on Deep Venous Thrombosis/Pulmonary Embolism: Current diagnosis of venous thromboembolism in primary care: a clinical practice guideline from the American Academy of Family Physicians and the American College of Physicians. Ann Intern Med 2007, 20:454-458.

6. Grau E, Tenías JM, Soto MJ, Gutierrez MR, Lecumberri R, Pérez JL, Tiberio G, RIETE Investigators: D-dimer levels correlate with mortality in patients with acute pulmonary embolism: Findings from the RIETE registry. Crit Care Med 2007, 35:1937-1941.

7. Panacek EA, Marshall JC, Albertson TE, Johnson DH, Johnson S, MacArthur RD, Miller M, Barchuk WT, Fischkoff S, Kaul M, Teoh L, Van Meter L, Daum L, Lemeshow S, Hicklin G, Doig C, Monoclonal Anti-TNF: a Randomized Controlled Sepsis Study Investigators: Efficacy and safety of the monoclonal anti-tumor necrosis factor antibody $F\left(a b^{\prime}\right) 2$ fragment afelimonmab in patients with severe sepsis and elevated interleukin-6 levels. Crit Care Med 2004, 32:2173-2182.

8. Dellinger RP, Carlet JM, Masur $\mathrm{H}$, Gerlach $\mathrm{H}$, Calandra $\mathrm{T}$, Cohen J, Gea-Banacloche J, Keh D, Marshall JC, Parker MM, Ramsay G, Zimmerman JL, Vincent JL, Levy MM, Surviving Sepsis Campaign Management Guidelines Committee: Surviving sepsis campaign guidelines for management of severs sepsis and septic shock. Crit Care Med 2004, 32:858-873.

9. Levy MM, Fink MP, Marshall JC, Abraham E, Angus D, Cook D, Cohen J, Opal SM, Vincent JL, Ramsay G, SCCM/ESICM/ACCP/ ATS/SIS: 2001 SCCM/ESICM/ACCP/ATS/SIS International Sepsis Definitions Conference. Crit Care Med 2003, 31:1250-1256.

10. Griffin JH, Fernandez JA, Gale AJ, Mosnier LO: Activated protein C. J Thromb Haemost 2007:73-80.

11. Mosnier LO, Zlokovic BV, Griffin JH: The cytoprotective protein C pathway. Blood 2007, 109:3161-3172.

12. Gierer $P$, Hoffmann JN, Mahr F, Menger MD, Mittlmeier T, Gradl G, Vollmar B: Activated protein $C$ reduces tissue hypoxia, inflammation, and apoptosis in traumatized skeletal muscle during endotoxemia. Crit Care Med 2007, 35:1966-1971.

13. Franscine N, Bachli EB, Blau N Leikaurf MS, Schaffner A, Schoedon G: Gene expression profiling of inflamed human endothelial cells and influence of activated protein C. Circulation 2004, 10:2903-2909.

14. Griffin JH, Fernández JA, Mosnier LO, Liu D, Cheng T, Guo H, Zlokovic BV: The promise of protein C. Blood Cells Mol Dis 2006, 36:211-216

15. Bernard GR, Vincent JL, Laterre PF, LaRosa SP, Dhainaut JF, Lopez-Rodriguez A, Steingrub JS, Garber GE, Helterbrand JD, Ely EW, Fisher CJ Jr, Recombinant human protein C Worldwide Eval- uation in Severe Sepsis PROWESS) study group: Efficacy and safety of recombinant activated protein $\mathrm{C}$ for severe sepsis. $N$ Engl J Med 2001, 344:699-709.

16. Sheth SB, Carvalho AC: Protein $\mathbf{S}$ and $C$ alterations in acutely ill patients. Am J Hematol 1999, 36:14-19.

17. Yan SB, Helterbrand JD, Hartman DL, Wright TJ, Bernard GD: Low levels of protein $C$ are associated with poor outcome in severe sepsis. Chest 2001, 120:915-922.

18. Kinasewitz GT, Yan SB, Basson B, Comp P, Russell JA, Cariou A, Um SL, Utterback B, Laterre PF, Dhainaut JF, PROWESS Sepsis Study Group: Universal changes in biomarkers of coagulation and inflammation occur in patients with severe sepsis, regardless of causative micro-organism. Crit Care 2004, 8:R82-R90.

19. Fourrier F, Chopin C, Goudemand J, Hendrycx S, Caron C, Rime A, Marey A, Lestavel P: Septic shock, multiple organ failure, and disseminated intravascular coagulation: compared patterns of antithrombin III, Protein $\mathbf{C}$ and protein $\mathbf{S}$ deficiencies. Chest 1992, 101:816-823.

20. Lorente JA, García-Frade LJ, Landín L, de Pablo R, Torrado C, Renes E, Garcia-Avello A: Time course of hemostatic abnormalities in sepsis and its relation to outcome. Chest 1993, 103:1536-1542

21. Mesters RM, Helterbrand J, Utterback BG, Yan B, Chao YB, Fernandez JA, Griffin JH, Hartman DL: Prognostic value of protein $\mathrm{C}$ concentrations in neutropenic patients at high risk of severe septic complications. Crit Care Med 2000, 28:2209-2216.

22. Macias WL, Nelson DR: Severe protein $C$ deficiency predicts early death in severe sepsis. Crit Care Med 2004:S223-S228.

23. Ely EW, Laterre PF, Angus DC, Helterbrand JD, Levy H, Dhainaut JF, Vincent JL, Macias WL, Bernard GR, PROWESS Investigators: Drotrecogin alfa (activated) administration across clinically important subgroups of patients with severe sepsis. Crit Care Med 2003, 31:12-19.

24. Peters M, Fijnvandraat K, Derkx B, Stassen M, Van Deventer SJH: Severely reduced protein $C$ levels predict a high mortality in meningococcal shock [abstract]. Thromb Haemost 1993, 69:A2337.

25. Hazelzet JA, Van der Voort E, Lindemans J, Ter Heerdt PGJ, Neijens $\mathrm{HJ}$ : Relation between cytokines and routine laboratory data in children with septic shock and purpura. Intensive Care Med 1994, 20:371-374.

26. Fijnvandraat K, Derkx B, Peters M, Bijlmer R, Sturk A, Prins MH, van Deventer SJ, ten Cate JW: Coagulation activation and tissue necrosis in meningococcal septic shock: severely reduced protein $\mathbf{C}$ levels predict a high mortality. Thromb Haemost 1995, 73:15-20.

27. Brandtzaeg P, Sandset PM, Joo GB, Ovstebo R, Abildgaard U, Kieruef $P$ : The quantitative association of plasma endotoxin, antithrombin, protein $\mathrm{C}$, extrinsic pathway inhibitor, and fibrinopeptide A in systemic meningococcal disease. Thromb Res 1989, 55:459-470.

28. Philippe J, Offner F, Leroux-Roels G, Vogelaers D, Baele G: Plasminogen activator inhibitor-1, protein $C$, antithrombin III and tissue plasminogen activator activities in the early phase of septic shock [abstract]. Thromb Haemost 1989, 65:A1836.

29. Hesselvik JF, Malm J, Dahlback B, Blomback M: Protein C, protein $\mathrm{S}$ and $\mathrm{C} 4 \mathrm{bbinding}$ protein in severe infection and septic shock. Thromb Haemost 1991, 65:126-129.

30. Leclerc F, Hazelzet J, Jude B, Hofhuis W, Hue V, Martinot A, Van der Voort $E$ : Protein $\mathbf{C}$ and $\mathbf{S}$ deficiency in severe infectious purpura of children: a collaborative study of $\mathbf{4 0}$ cases. Intensive Care Med 1992, 18:202-205.

31. Román J, Velasco F, Fernandez F, Fernandez M, Villalba R, Rubio $\mathrm{V}$, Torres A: Protein C, protein S, and C4b-binding protein in neonatal severe infection and septic shock. J Perinat Med 1992, 20:111-116.

32. Piette WW, Shasby DM, Kealey GP, Olson JD: Retiform purpura is a sign of severe acquired protein $C$ deficiency and risk of progression to purpura fulminans in sepsis and disseminated intravascular coagulation [abstract]. Clin Res 1993, 41:A253.

33. Powars D, Larsen R, Johnson J, Hulbert T, Sun T, Patch MJ, Francis $\mathrm{R}$, Chan L: Epidemic meningococcemia and purpura fulminans with induced protein C deficiency. Clin Infect Dis 1993, 17:254-261.

34. Shorr AF, Bernard GR, Dhainaut JF, Russell JR, Macias WL, Nelson DR, Sundin DP: Protein C concentrations in severe sepsis: 
an early directional change in plasma levels predicts outcome. Crit Care 2006, 10:R92.

35. Vincent JL, Bernard GR, Beale R, Doig C, Putensen C, Dhainaut $\mathrm{JF}$, Artigas A, Fumagalli R, Macias W, Wright T, Wong K, Sundin DP, Turlo MA, Janes J: Drotrecogin alfa (activated) treatment in severe sepsis from the global open-label trial ENHANCE: further evidence for survival and safety and implications for early treatment. Crit Care Med 2005, 33:2266-2277.

36. Li Z, Meredith MP, Hoseyni MS: A method to assess the proportion of treatment effect explained by a surrogate endpoint. Stat Med 2001, 20:3175-3188.

37. Remick DG: Pathophysiology of sepsis. Am r J Pathol 2007, 170:1435-1444.

38. Hawker F: Liver dysfunction in critical illness. Anaesth Intensive Care 1991, 19:165-181.

39. Geier A, Fickert P, Trauner M: Mechanisms of disease: mechanisms and clinical implications of cholestasis in sepsis. Nat Clin Pract Gastroenterol Hepato/ 2006, 3:574-585.

40. De Gruttola VG, Clax P, DeMets DL, Downing GJ, Ellenberg SS, Friedman L, Gail MH, Prentice R, Wittes J, Zeger SL: Considerations in the evaluation of surrogate endpoints in clinical trials: summary of a National Institutes of Health Workshop. Contro/led Clin Trials 2001, 22:485-502.

41. Freedman LS, Graubard BI, Schatzkin A: Statistical validation of intermediate endpoints for chronic diseases. Stat Med 1992, 11:167-178

42. Vangerow B, Shorr AF, Wyncoll D, Janes J, Nelson D, Reinhart K: The Protein C pathway: implications for the design of the RESPOND study. Crit Care 2007:S4. 